\title{
Modelling Multicomponent Droplet and Spray Evaporation Dynamics using Functional Group Method UNIFAC
}

\author{
W. Yang ${ }^{1}$, J. Xia $^{* 1}$ \\ ${ }^{1}$ Department of Mechanical and Aerospace Engineering \& Institute of Energy Futures, \\ Brunel University London, Uxbridge UB8 3PH, UK \\ *Corresponding author email: jun.xia@brunel.ac.uk
}

\begin{abstract}
In a multicomponent miscible liquid mixture, structural interactions among components distinctly different in chemical structure and molecular size affect evaporation. To account for these effects, the UNIFAC model [1, 2] has been used to determine the activity coefficient, accounting for the non-ideality of vapour-liquid equilibrium (VLE). The phase diagram of an ethanol/isooctane mixture determined by using the UNIFAC model shows good agreement with measurement. For an ethanol/isooctane droplet, non-ideal VLE models which correct the activity of liquid components must be used to properly predict the evaporation, when ethanol is the predominantly major component (e.g. 78 vol.\% of ethanol - E78 considered in this study). The separation factor, which quantifies the relative volatility between two components, directly shows the heavier hydrocarbon component isooctane evaporates faster compared to ethanol for the E78 droplet during the whole droplet life time. With UNIFAC, the evaporation dynamics of a two-component isooctane/ethanol and a four-component gasoline/ethanol spray is then investigated and compared to predictions using the ideal Raoult's law. Compared to other approaches, the group contribution method UNIFAC only requires properties of constituent functional groups of each component, which is particularly useful when no VLE data is available for binary-component subsystems of the multi or many-component mixture.
\end{abstract}

\section{Keywords}

Multicomponent; Evaporation; Droplet; Spray; UNIFAC

\section{Introduction}

Since renewable biofuel is now widely used to reduce carbon emissions and improve fuel efficiency, gasoline and its mixture with biofuel (e.g. ethanol) are directly injected into the combustion chamber in modern gasoline direct-injection engines using smart fuel-injection strategies. Properly modelling evaporation of a multicomponent (e.g. gasoline/ethanol) spray therefore becomes essential since a biofuel mixture usually consists of not only alkanes but also alcohols and dimethyl, which are distinctly different in chemical structure and molecular size. The structurally dissimilar components behave rather differently during the evaporation process, and structural interaction inside the mixture that affects evaporation cannot be ignored $[3,4,5]$.

With the shell and ideal-mixture models [6], liquid hydrocarbon components evaporate according to their relative volatilities, in a way similar to batch distillation. Ra et al. [7] developed a vaporisation model based on Raoult's law for multicomponent fuels as surrogates for gasoline and diesel. It was found that light-end components of the mixture appear upstream of the spray plume, whereas heavy-end components predominantly appear near the tip of the spray. Ebrahimian and Habchi [8] proposed a multicomponent droplet evaporation model at all pressure conditions. The vapour mole fractions at the droplet surface are determined by VLE conditions, in which fugacity coefficients of the vapour and liquid phases are determined by a two-parameter cubic equation of state, taking into account high-pressure correction. A detailed study has been conducted on two-component liquid-mixture evaporation for both droplets [3] and sprays [4] under spark-ignition engine operating conditions. Non-ideal effects of the mixture, modelled by the Non Random Two Liquid (NRTL) approach [2] in [3] and [4], were found 
to significantly affect the prediction of vapour formation and distribution. Kitano et al. [9] investigated droplet evaporation and combustion of single- and multicomponent surrogates for Jet-A. The modified Raoult's law incorporated non-equilibrium thermodynamic effects.

The objective of the present work is to investigate effects of non-ideal vapour-liquid equilibrium models on evaporation dynamics of multicomponent ethanol/gasoline droplets and sprays. The activity coefficients, which correct liquid-component activities towards a more realistic vapour-liquid equilibrium at the gas/liquid interface (droplet surface), are estimated by the UNIFAC model [1, 2]. UNIFAC has been implemented into ANSYS Fluent, and evaporation characteristics of a multicomponent droplet and spray are compared between the ideal Raoult's law and non-ideal UNIFAC model using the Eulerian/Lagrangian modelling framework for gasliquid two-phase flow of Fluent. The biofuel/gasoline fuel blend is approximated first by a twocomponent ethanol/isooctane mixture. A ternary mixture is then used as a more realistic surrogate for gasoline, and evaporation dynamics of a four-component ethanol/gasoline spray is compared between the ideal and non-ideal VLE models.

The discrete component model concept [7] has been used in the present study. Using zero-dimensional (0D) models, droplets are not identified with the grid resolution used. For modelling evaporation of a single droplet, it would be interesting to investigate how non-uniform distributions of droplet temperature and liquid-species mass fractions and densities, including one-dimensional and multidimensional distributions suitable for quiescent environment or convective heating, respectively, affect droplet evaporation, which can then benchmark and improve 0D models. For spray evaporation, the Eulerian-Lagrangian methodology has been widely used, as in the present study, in which droplets are traced as point sources in Cartesian grids for the gas phase. In such a configuration, deploying grids inside a droplet will not bring significant benefits, even if computational costs are not a concern, because the resolution of the boundary conditions for a point-source droplet at each time step are determined by the local Eulerian grids. If droplets are to be identified with grids to incorporate into the modelling non-uniform liquid-property distributions inside a droplet, then (simplified) interface capturing or tracking techniques which include mass and energy transfer across gas-liquid interfaces should be used instead.

\section{Evaporation model}

For a miscible multicomponent fuel droplet, the evaporation rate $\dot{m}_{\mathrm{d}, i}\left[\mathrm{SI}\right.$ unit: $\left.\mathrm{kg} \cdot \mathrm{s}^{-1}\right]$ of component $i$ in the liquid mixture can be determined by

$$
\dot{m}_{\mathrm{d}, i}=k_{\mathrm{c}, i} A_{\mathrm{d}} \rho_{\mathrm{g}} \ln \left(1+B_{\mathrm{m}, i}\right),
$$

where $A_{\mathrm{d}}\left[\mathrm{m}^{2}\right]$ is the droplet surface area; $\rho_{\mathrm{g}}\left[\mathrm{kg} \cdot \mathrm{m}^{-3}\right]$ is the density of the ambient gas; $k_{\mathrm{c}, i}$ $\left[\mathrm{m} \cdot \mathrm{s}^{-1}\right]$ is the mass transfer coefficient of component $i$.

The mass transfer Spalding number $B_{\mathrm{m}, i}[-]$ of component $i$ can be calculated as $B_{\mathrm{m}, i}=$ $\left(Y_{i, \mathrm{~V}}-Y_{i, \mathrm{~g}}\right) /\left(\varepsilon_{i}-Y_{i, \mathrm{~V}}\right)$, with $Y_{i, \mathrm{~V}}[-]$ denoting the mass fraction of vapour component $i$ at the droplet surface and $\varepsilon_{i}$ the mass fraction of vapour component $i$ in the fuel vapour: $\varepsilon_{i}=$ $Y_{i, \mathrm{~V}} / \Sigma_{i} Y_{i, \mathrm{~V}}$. It is clear that the Spalding number for each component is crucial for the accuracy of the evaporation model and a key quantity is the vapour mass fraction $Y_{i, \mathrm{~V}}$ of each component at the droplet surface, which is related to the vapour mole fraction $X_{i, \mathrm{~V}}[-]$ at the droplet surface through $Y_{i, \mathrm{~V}}=X_{i, \mathrm{~V}} M_{i} / M_{\mathrm{m}, \mathrm{V}}$, where $M_{i}\left[\mathrm{~kg} \cdot \mathrm{mol}^{-1}\right]$ is the molecular mass of component $i$, and $M_{\mathrm{m}, \mathrm{V}}\left[\mathrm{kg} \cdot \mathrm{mol}^{-1}\right]$ is the mean molecular mass of the vapour mixture at the droplet surface.

Within the scope of equilibrium thermodynamics, the VLE model widely adopted by most of the computational studies of multicomponent droplet and spray evaporation, especially by the spray and combustion communities, is Raoult's law $[6,10]$

$$
X_{i, \mathrm{~V}}=X_{i, \mathrm{~L}} \frac{P_{\mathrm{sat}, i}}{P} .
$$

The ratio between the mole fractions of component $i$ in the gas and liquid phases at the interface is therefore fully determined by the vapour saturation pressure $P_{\mathrm{sat}, i}[\mathrm{~Pa}]$ of that (pure) 
component at the local $T[\mathrm{~K}]$ and $P$.

A generalised VLE model satisfies the condition that the fugacity $f$ (a corrected pressure) $[\mathrm{Pa}]$ of the vapour is in equilibrium with that of the liquid for each component at the gas/liquid interface, i.e. $f_{i, \mathrm{~L}}=f_{i, \mathrm{~V}}$. For an ideal gas mixture, the vapour mole fraction $X_{i, \mathrm{~V}}$ of component $i$ can then be determined by [2]

$$
X_{i, \mathrm{~V}}=\gamma_{i} X_{i, \mathrm{~L}} \frac{P_{\mathrm{sat}, i}}{P},
$$

if the total pressure $P$ is sufficiently low. $\gamma_{i}[-]$ is the activity coefficient of component $i$ in the liquid phase.

The activity coefficient $\gamma_{i}$ is therefore an additional key quantity determining the evaporation characteristics of component $i$ compared to Eq. 2. For an ideal liquid mixture, $\gamma_{i}=1 \forall i$ and the generalised VLE model Eq. 3 reduces to Raoult's law Eq. 2. However, when structural dissimilarity and microscopic interaction between components become significant, activity coefficients should be introduced to account for the deviation of the evaporation dynamics of a multicomponent liquid mixture from that predicted by the ideal VLE model Raoult's law.

In the present study, activity coefficients are determined by the UNIFAC (UNIversal quasichemical Functional group Activity Coefficients) model [1]. UNIFAC is a group contribution method, in which a molecule is regarded as an aggregate of functional groups to correlate thermodynamic properties. A physical property of a fluid is assumed to be the sum of contributions made by the molecule's functional groups instead of the molecules themselves. The properties of a very large number of multicomponent liquid mixtures can therefore be correlated in terms of a much smaller number of parameters which characterise the contributions of individual groups [2].

Since it only requires the data of functional groups of each component in the mixture, which are published and validated, the UNIFAC model is able to determine activity coefficients when there is no VLE data available for any of the binary-component subsystems of the multicomponent mixture. The interaction parameters between functional groups are estimated base on experimental phase equilibrium data. Note experimental VLE data of all the binary-component subsystems are always needed in other approaches to determining activity coefficients that are based on interactions between components (molecules) but not functional groups.

When different functional groups exist in a liquid mixture, net intermolecular forces arise due to structural differences. Under these forces, the activity coefficients could considerably deviate from unity and the mixture evaporation can no longer be approximated by an ideal VLE model. Although Raoult's law can be reasonably used for modelling evaporation of gasoline, which is a hydrocarbon mixture, it becomes necessarily crucial to consider activity coefficients if aromatic species interact with paraffinic ones in the mixture [4]. In addition, the degree of non-ideality can sharply increase by adding functional groups of ethanol into the hydrocarbon mixture. The high polarity of the functional group causes an increase of intermolecular forces and increases the excess Gibbs energy $G^{\mathrm{E}}$. Therefore for a gasoline/ethanol liquid-fuel mixture considered in the present study, activity coefficients must be considered to properly model the evaporation dynamics of the multicomponent liquid based on the VLE concept, as will be shown later.

\section{Results and discussion}

Activity coefficient Figure 1 shows the activity coefficients of ethanol and isooctane in a binary-component liquid mixture at the liquid-phase temperature $T_{\mathrm{L}}=300 \mathrm{~K}$ predicted by the UNIFAC model. The abscissa is the liquid-phase mole fraction $X_{\mathrm{C}_{8} \mathrm{H}_{18}, \mathrm{~L}}$ of isooctane. An evident trend of both $\gamma_{\mathrm{C}_{8} \mathrm{H}_{18}}$ and $\gamma_{\mathrm{C}_{2} \mathrm{H}_{5} \mathrm{OH}}$ is when the component becomes the minor one in the liquid mixture, especially as $X_{\mathrm{C}_{2} \mathrm{H}_{5} \mathrm{OH}, \mathrm{L}} \rightarrow 0$ or $X_{\mathrm{C}_{8} \mathrm{H}_{18}, \mathrm{~L}} \rightarrow 0$, its activity coefficient rapidly grows, demonstrating its evaporation characteristics deviate considerably from Raoult's law in which its $\gamma$ is always 1. Even as the heavier, less "volatile" component in the mixture under the initial condition, it is very possible that isooctane will become more active in the liquid and 


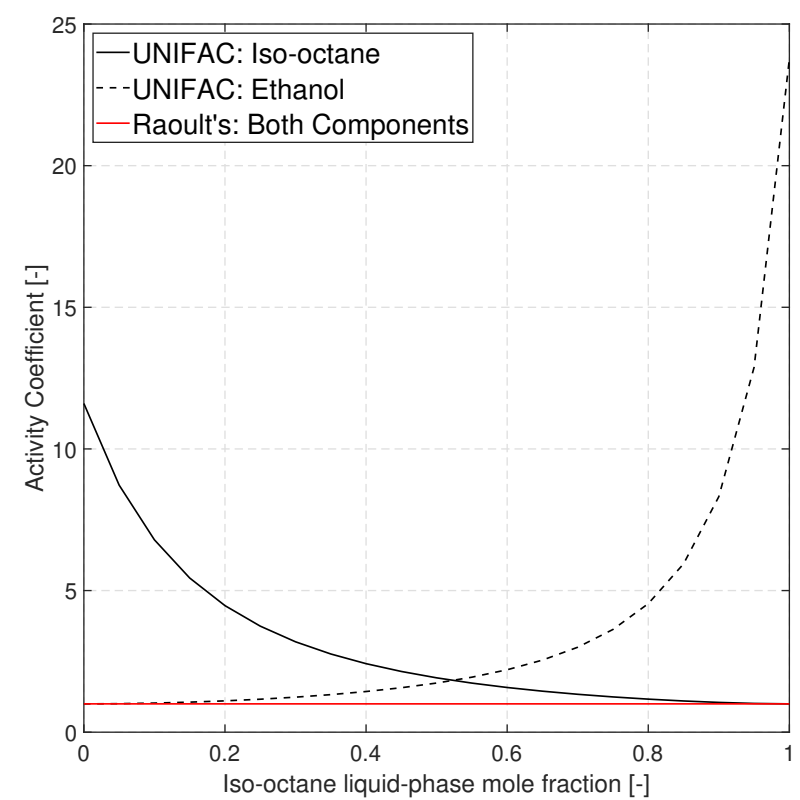

Figure 1. Activity coefficients $\gamma$ of ethanol and isooctane for a binary-component ethanol/isooctane mixture at $T_{\mathrm{L}}=T_{\mathrm{d}}=300 \mathrm{~K}$

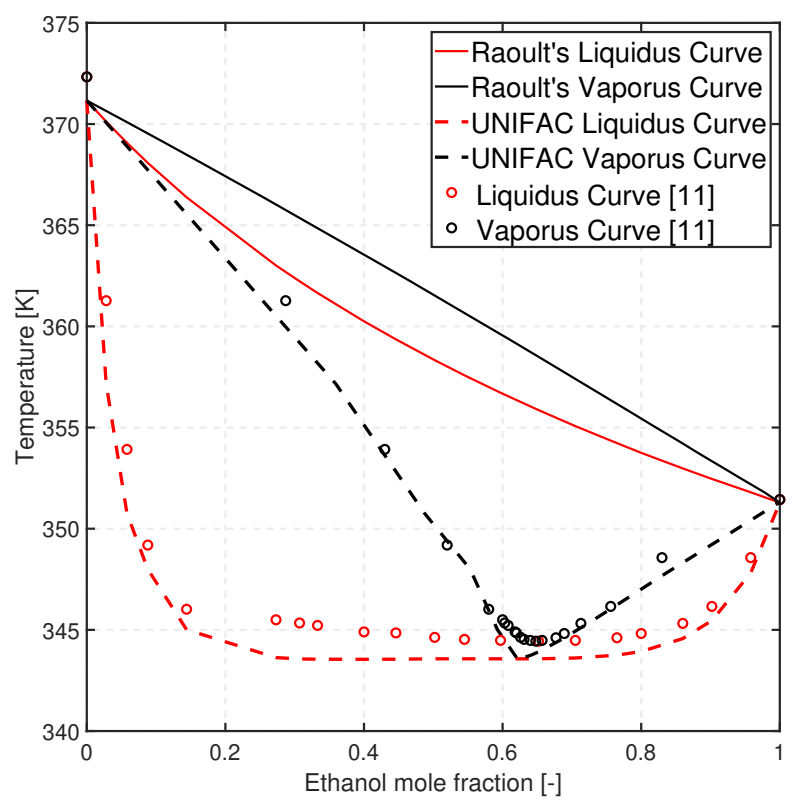

Figure 2. Ethanol/isooctane phase diagram at 1 bar.

evaporate faster at the droplet surface. When ethanol is the minor component, the escape of ethanol molecules at the liquid-gas interface will also be enhanced due to its activity in the liquid mixture corrected by $\gamma_{\mathrm{C}_{2} \mathrm{H}_{5} \mathrm{OH}}\left(\gamma_{\mathrm{C}_{2} \mathrm{H}_{5} \mathrm{OH}}>5\right.$ as $\left.X_{\mathrm{C}_{2} \mathrm{H}_{5} \mathrm{OH}, \mathrm{L}}<20 \%\right)$.

Phase Diagram The temperature-composition phase diagrams $T-X_{\mathrm{C}_{2} \mathrm{H}_{5} \mathrm{OH}, \mathrm{L}}-X_{\mathrm{C}_{2} \mathrm{H}_{5} \mathrm{OH}, \mathrm{V}}$ of the binary-component ethanol/isooctane mixture at $P=1$ bar determined by Raoult's law and UNIFAC are illustrated in Fig. 2 and compared to published data [11]. It can be seen that good agreement between UNIFAC and [11] has been achieved, including the minimum-boiling azeotrope. The two-phase coexisting region predicted by Raoult's law completely mismatches those predicted by UNIFAC. Also with Raoult's law, since the liquidus curve always locates on the left side of the vaporus one, $X_{\mathrm{C}_{2} \mathrm{H}_{5} \mathrm{OH}, \mathrm{L}}<X_{\mathrm{C}_{2} \mathrm{H}_{5} \mathrm{OH}, \mathrm{V}}$ at any valid temperature in the whole composition space. But experimental data [11] have shown a different trend, namely, the relation of the magnitudes of the liquid- and vapour-phase mole fractions of ethanol is separated by the azeotrope point, on the left side of which $X_{\mathrm{C}_{2} \mathrm{H}_{5} \mathrm{OH}, \mathrm{L}}<X_{\mathrm{C}_{2} \mathrm{H}_{5} \mathrm{OH}, \mathrm{V}}$ and on the right side of which $X_{\mathrm{C}_{2} \mathrm{H}_{5} \mathrm{OH}, \mathrm{L}}>X_{\mathrm{C}_{2} \mathrm{H}_{5} \mathrm{OH}, \mathrm{V}}$, as reproduced by UNIFAC.

Droplet evaporation The evaporation of an ethanol/isooctane droplet in quiescent nitrogen is simulated by using the lumped OD model. The initial droplet diameter and temperature are $D_{\mathrm{d}, 0}$ $=100 \mu \mathrm{m}$ and $T_{\mathrm{d}, 0}=25{ }^{\circ} \mathrm{C}$, respectively. The ambient temperature and pressure are $600{ }^{\circ} \mathrm{C}$ and 10 bar, respectively, for comparison with published data. The initial fuel compositions are E36 and E78, i.e. 36 and 78 vol.\% ethanol in the liquid mixture. The corresponding mole and mass fractions of ethanol in the E36 liquid-fuel are $61.3 \%$ and $39 \%$, respectively; and $90.83 \%$ and $80 \%$, respectively, for E78. The predictions using UNIFAC, Raoult's law (indicated as the ideal model) and NRTL [3] have been compared.

Time evolutions of the squared normalised droplet diameter $D_{\mathrm{d}}^{2} / D_{\mathrm{d}, 0}^{2}$ and the droplet surface temperature $T_{\mathrm{d}, s}$ are illustrated in Figs. 3(a) and 3(b), respectively. The E78 droplet has a slower evaporation process and thus a longer droplet life time than the E36 one. The three models produce quantitatively similar results except at the final stage. For E36, the droplet life time predicted by the NRTL model is slightly longer than the other two models. For E78, the droplet life time predicted by Raoult's law is slightly shorter than the other two models.

As shown in Fig. 3(b), the (surface) temperature of the E36 droplet predicted by Raoult's law monotonically increases with time, with a steeper and a smaller slope at the initial and later stages, respectively. Compared to Raoult's law, following an initial rapid increase of the droplet 


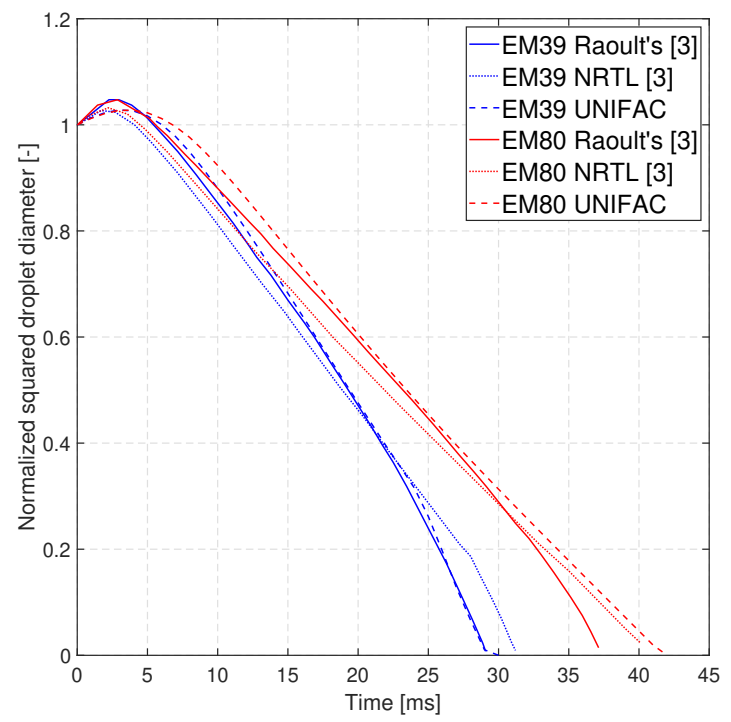

(a) Droplet diameter

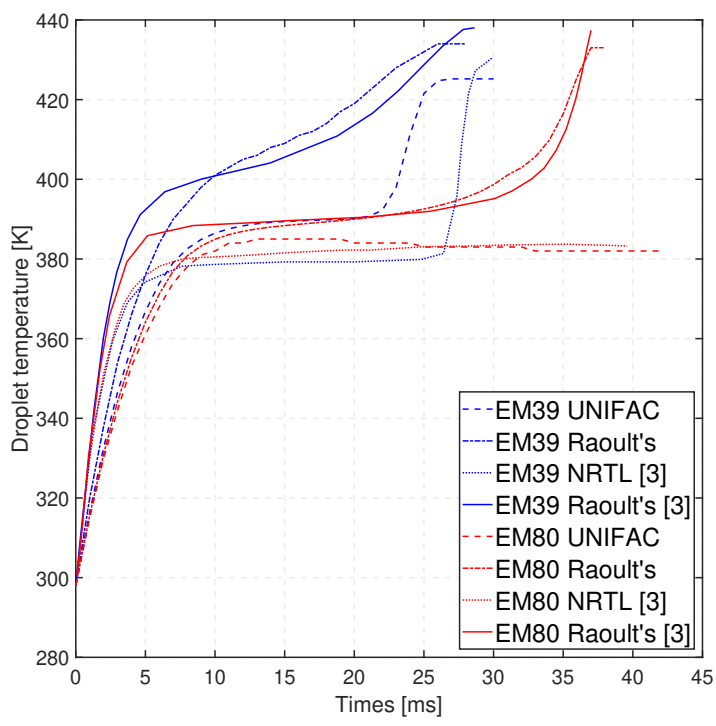

(b) Droplet temperature

Figure 3. Droplet evaporation statistics. E36=EM39; E78=EM80. EM: ethanol mass fraction.

temperature, a steady droplet temperature is reached with UNIFAC. Then a sharp increase of the droplet temperature is seen, which occurs at $t \approx 24 \mathrm{~ms}$, when ethanol in the droplet completes evaporation. NRTL in [3] predicts a later time when ethanol completes evaporation. For the E78 droplet, both UNIFAC and NRTL predict $T_{\mathrm{d}}$ almost remains at $\sim 380 \mathrm{~K}$ until the end of the droplet life time. With Raoult's law, the droplet temperature rapidly rises at the final stage of the evaporation after $t \approx 30 \mathrm{~ms}$. The ratio between the isooctane and ethanol mass of the droplet predicted by Raoult's law shows a trend of sharp increase after $t \approx 30 \mathrm{~ms}$ (not shown), as the minor component isooctane is wrongly predicted to gradually become the absolutely major component of the droplet. With UNIFAC the ratio decreases towards 0 , i.e. ethanol is always the absolutely major component of the liquid mixture during the evaporation and isooctane completes evaporation first.

Vapour mole fractions $X_{i, \mathrm{~V}}$ at the droplet surface are a key quantity that can further show the difference between an ideal and a more realistic VLE model. Figures 4 shows $X_{i, \mathrm{~V}}$ of the E78 mixture in a 10-based logarithmic scale against a non-dimensional time normalised by the droplet life time. While Raoult's law can predicts the correct trends, but different quantitative details, of the evaporation of the E36 mixture (not shown), the same cannot be said for the E78 mixture. In Fig. 4, both the UNIFAC and NRTL models predict $X_{\mathrm{C}_{8} \mathrm{H}_{18}, \mathrm{~V}}$ will continuously decrease after an initial rise immediately after the droplet evaporation starts. Raoult's law, on the other hand, predicts $X_{\mathrm{C}_{8} \mathrm{H}_{18}, \mathrm{~V}}$ will monotonically increase during the evaporation. In addition, it predicts that $X_{\mathrm{C}_{2} \mathrm{H}_{5} \mathrm{OH}, \mathrm{V}}$ will rapidly drop in the final stage, a trend not revealed by the more realistic, non-ideal UNIFAC and NRTL models. It is clear that to only compare the $D^{2}$ law is not sufficient to show that the droplet evaporation has been properly modelled, although this practice has been widely adopted by the community.

The separation factor $\alpha_{i j}$ measures the relative volatility between two components $i$ and $j$ or which of the two components vaporises faster at the gas-liquid interface (droplet surface) [3]. Since in Raoult's law, $\alpha_{i j}$ depends only on saturation vapour pressures of the pure components, i.e. $\alpha_{i j}=P_{\text {sat }, i} / P_{\text {sat }, j}=f(T)$, which is a function of temperature. Since saturation vapour pressure monotonically increases with temperature, $\alpha_{i j}$ will not undergo transition through 1 from less-than-1 to higher-than-1, or vice versa, during the evaporation. Equivalently speaking, the relative volatility of respective components in a multicomponent liquid mixture will remain identical to what the initial condition dictates throughout the evaporation process. On the other hand, for more realistic VLE models such as UNIFAC and NRTL, $\alpha_{i j}=\gamma_{i} P_{\text {sat }, i} /\left(\gamma_{j} P_{\text {sat }, j}\right)=$ $f\left(T, X_{i, \mathrm{~L}}, P\right)$, which is a function of temperature, pressure and liquid-fuel composition. The 


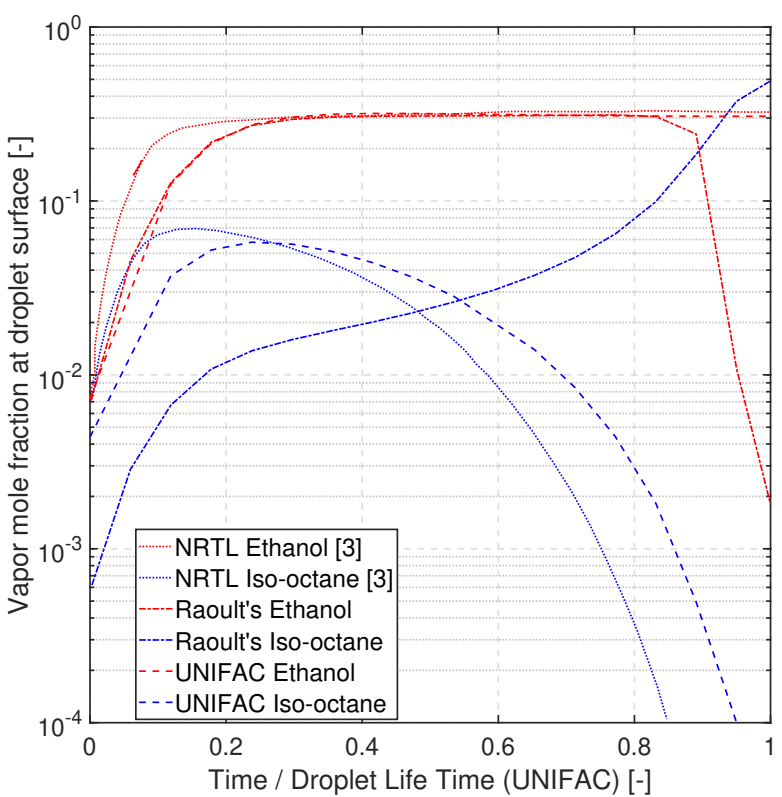

Figure 4. Vapour-phase mole fractions at droplet surface: E78/EM80.

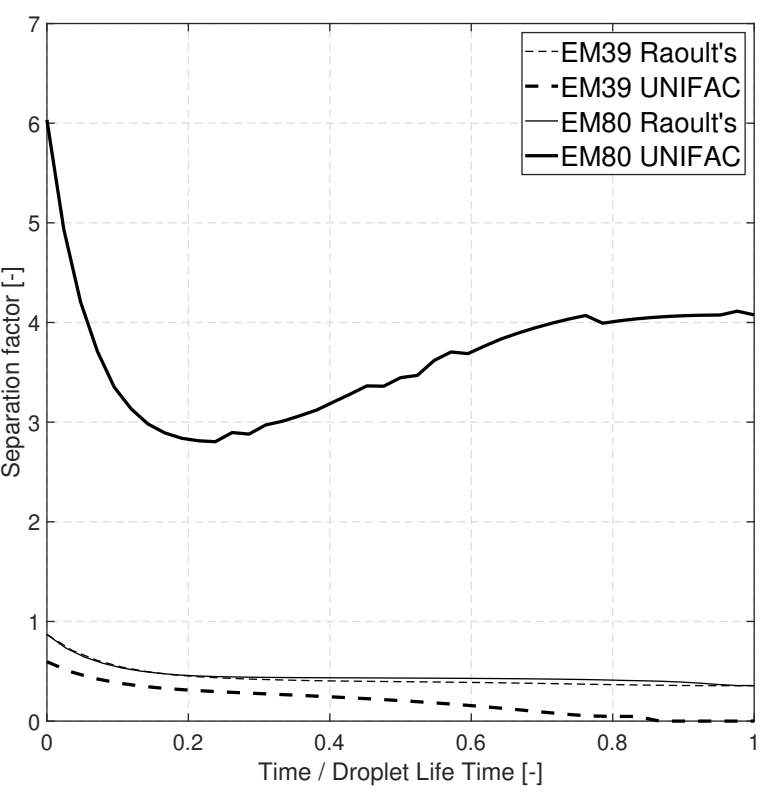

Figure 5. Separation factor during binary-component droplet evaporation, defined by the relative volatility of isooctane compared to ethanol, i.e. if $\alpha_{\mathrm{C}_{8} \mathrm{H}_{18}, \mathrm{C}_{2} \mathrm{H}_{5} \mathrm{OH}}>1$, isooctane vaporises faster than ethanol.

relative volatility among different components could switch between the two regimes, i.e. $\alpha_{i j}>$ 1 and $\alpha_{i j}<1$, during the evaporation and does not depend only on initial conditions [3]. It is clear that $\alpha_{i j}$ and thus $\gamma_{i}$ is the important mechanism which is missing in Raoult's law.

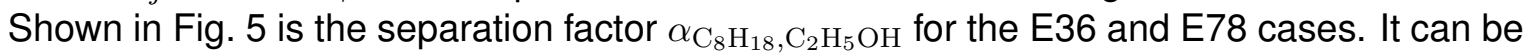
seen that for the E36 case, Raoult's law presents a correct trend of the relative volatility between the two components, although UNIFAC gives a lower separation factor throughout the droplet evaporation. While for the E78 case, since the separation factor predicted by Raoult's law depends exclusively on vapour pressure, $\alpha_{\mathrm{C}_{8} \mathrm{H}_{18}, \mathrm{C}_{2} \mathrm{H}_{5} \mathrm{OH}}$ is very close to that for the E36 case. While for UNIFAC, $\alpha_{\mathrm{C}_{8} \mathrm{H}_{18}, \mathrm{C}_{2} \mathrm{H}_{5} \mathrm{OH}}>1$ even under the initial condition and during the whole course of the evaporation process. In plain words, isooctane is the more volatile component and thus evaporates faster than ethanol in this case from the very beginning of evaporation, despite a higher saturation vapour pressure of ethanol.

In general when a more "volatile" component (ethanol in this case), only in terms of saturation vapour pressure, is the major component of the liquid mixture, especially when its volume fraction is considerably higher than that of the other component, Raoult's law will give misleading evaporation modelling results. With more realistic VLE models such as UNIFAC and NRTL taking into account the activity of the component and predicting a corrected liquid mole fraction by the activity coefficient $\gamma_{i}$, the separation factor $\alpha_{12}$ is able to properly predict the regime of the relative volatility between two components, which can be transited during or even from the beginning of the evaporation.

Spray evaporation Spray simulations are performed with ANSYS Fluent using the EulerianLagrangian modelling framework, with the UNIFAC model implemented as a User Defined Function. A OD rapid internal mixing model [6] is used to approximate the dispersing liquid phase by assuming all the liquid components are well mixed and each droplet has a uniform temperature and species composition. The $k-\varepsilon$ turbulence model is used to solve the turbulence kinetic energy $k$ and the turbulent dissipation rate $\varepsilon$. No breakup models were considered.

The SIMPLE algorithm for pressure-velocity coupling is used. The least squares cell-based method is used to compute spatial gradients, with a second-order scheme for pressure, secondorder upwind schemes for momentum, component species and energy, first-order upwind schemes for $k$ and $\varepsilon$, respectively. A first-order implicit scheme is used for time advancement 


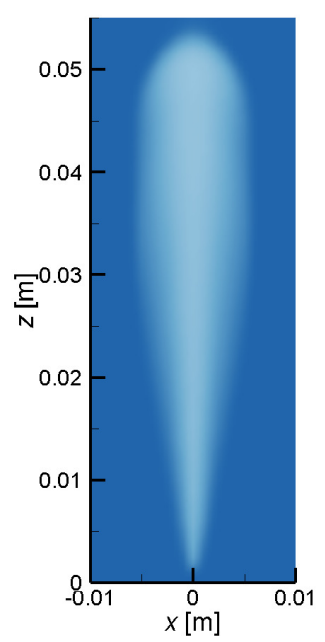

(a) Raoult's law

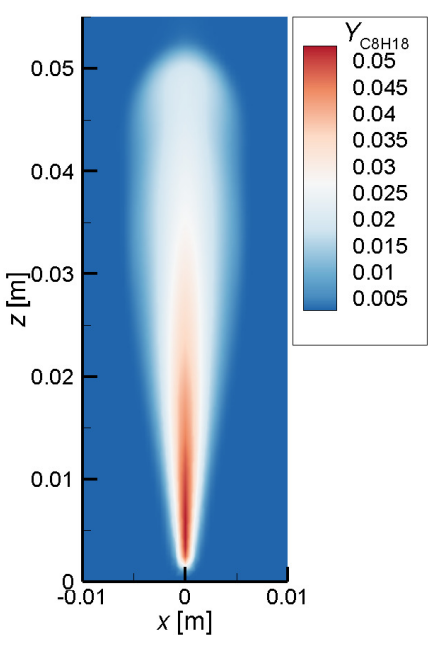

(b) UNIFAC

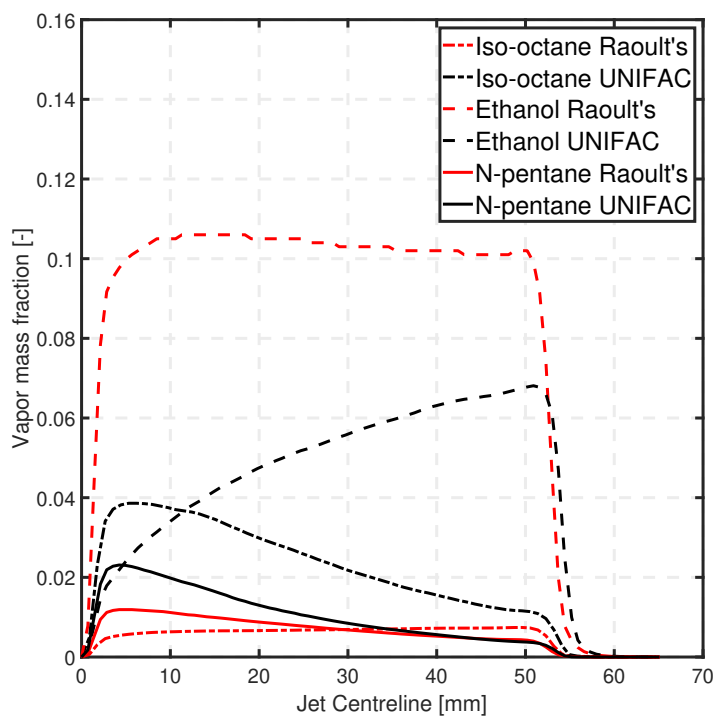

Figure 7. Vapour mass fractions of an E85, four-component ethanol/gasoline spray on jet centreline.

for the transient simulation.

The three-dimensional simulation domain is $20 \times 20 \times 70 \mathrm{~mm}^{3}$ and filled with inert quiescent nitrogen initially. The ambient temperature and pressure are $T=473 \mathrm{~K}$ and $P=0.56 \mathrm{MPa}$, respectively. At the nozzle inlet, the injection pressure of the spray is $20 \mathrm{MPa}$. The injection velocity is $224 \mathrm{~m} / \mathrm{s}$, which is determined by the Bernoulli equation, and the injection duration is $1 \mathrm{~ms}$. The initial sizes of the injected Lagrangian parcels (sprayed droplets) follow a RosinRammler distribution with a mean droplet size of $9 \mu \mathrm{m}$. The initial temperature of the discrete phase is $353 \mathrm{~K}$. Pressure outlet with 0 turbulent intensity is used at the four side boundaries and the downstream outlet of the domain. A cartesian mesh with a uniform grid spacing 0.4 $\mathrm{mm}$ in all the three directions is used.

The above setup and initial/boundary conditions [4] have been used for all spray simulations.

For the E85 ethanol/isooctane spray in which ethanol is the major component, the isooctane vapour distribution at $1 \mathrm{~ms}$ after the spray injection is shown in Fig. 6 . It can be seen that Raoult's law predicts a much slower evaporation process of isooctane and thus a much lower $Y_{\mathrm{C}_{8} \mathrm{H}_{18}, \mathrm{~V}}$. At $30 \mathrm{~mm}$ downstream of the nozzle, $Y_{\mathrm{C}_{8} \mathrm{H}_{18}, \mathrm{~V}}$ is slightly over 0.01 on the jet centreline with Raoult's law, but both UNIFAC and NRTL predict $Y_{\mathrm{C}_{8} \mathrm{H}_{18}, \mathrm{~V}} \approx 0.03$ (not shown).

Evaporation dynamics of a four-component gasoline/ethanol spray is simulated using the UNIFAC model and Raoult's law. A three-component mixture [12] has been used as a surrogate for gasoline, which consists of $n$-pentane, isooctane and $n$-decane with a mass fraction of $0.24,0.56,0.2$, respectively. The four-component fuel can achieve more realistic evaporation dynamics according to the distillation curve. An E85 spray is considered.

Figure 7 illustrates the vapour mass fractions of isooctane, $n$-pentane and ethanol along the spray jet centreline. For the major component ethanol, Raoult's law predicts $Y_{\mathrm{C}_{2} \mathrm{H}_{5} \mathrm{OH}, \mathrm{V}}$ rapidly rises to a steady value $\sim 0.1$ within a short distance at $\sim 10 \%$ of the spray tip. However, with UNIFAC, ethanol evaporates much more slowly. $Y_{\mathrm{C}_{2} \mathrm{H}_{5} \mathrm{OH}, \mathrm{V}}$ slowly increases on the centreline towards the spray tip, where its maximal value below 0.07 is reached, although it is much lower than the prediction by Raoult's law.

For the major component isooctane of the gasoline surrogate, the predictions by the two models are again very different. With UNIFAC, $Y_{\mathrm{C}_{8} \mathrm{H}_{18}, \mathrm{~V}}$ rapidly increases to its maximal value 0.04 in the vicinity of the nozzle, and then slowly decreases towards the spray tip at $Z \approx 55$ $\mathrm{mm}$. While for Raoult's law, $Y_{\mathrm{C}_{8} \mathrm{H}_{18}, \mathrm{~V}}$ continuously increases towards the spray tip, where its maximal value $<0.01$ is reached. For $n$-pentane, the trends of the vapour distribution are similar between the two models, with the maximal value of $Y_{n \mathrm{C}_{5} \mathrm{H}_{12}, \mathrm{~V}}$ predicted by UNIFAC twice that by Raoult's law. 
It should be noted that with UNIFAC, the vapour mass fraction of isooctane upstream near the nozzle $(Z<\sim 10 \mathrm{~mm})$ are higher than that of ethanol, despite a much higher volume and mass fraction of ethanol in the multicomponent liquid-fuel mixture, which indicates the evaporation rate of isooctane immediately following spray injection is much higher than that of ethanol, whereas the result modelled by Raoult's law leads to otherwise.

\section{Conclusions}

The significance of non-ideality effects on evaporation dynamics of a multicomponent liquid-fuel droplet and spray has been demonstrated in the present study. When component structures exhibit dissimilarity, e.g. between ethanol $\left(\mathrm{C}_{2} \mathrm{H}_{5} \mathrm{OH}\right)$ and isooctane $\left(\mathrm{C}_{8} \mathrm{H}_{18}\right)$, evaporation predicted by an ideal VLE model can be misled. The activity coefficient introduced in the UNIFAC model can help properly determine the phase equilibrium at the droplet surface, most importantly, the vapour phase mole fraction $X_{i, \mathrm{v}}$ of all components of the liquid mixture.

The comparisons between Raoult's law and UNIFAC on the phase diagram of the ethanolisooctane mixture and droplet/spray evaporation dynamics have shown the necessity and importance of considering a more realistic activity of liquid components. Specifically, although Raoult's law could reasonably predict the evaporation process of the two-component droplet when the lighter and thus intuitively more volatile component ethanol is the minor species, non-ideal VLE models must be used to properly predict the evaporation when ethanol is the predominant component, for which Raoult's law fails predictions on the droplet surface temperature, vapour concentrations on the droplet surface and the separation factor, even qualitatively.

The fact that UNIFAC is based on interactions among functional groups, but not components themselves, makes the model uniquely advantageous in extending its application towards many-component liquid-fuel mixtures, without the need of additional measurement for any binary-component subsystem if the corresponding VLE data are not available.

\section{Acknowledgements}

This paper is adapted from [13]. Financial support from MSCA-ITN-2019 under grant agreement No 861002 is gratefully acknowledged.

\section{References}

[1] Fredenslund, A., Jones, R. L., Prausnitz, J. M., 1975, AlChE Journal, 21, pp. 1086-1099.

[2] Reid, R. C., Prausnitz, J. M., Poling, B. E., 1987, "The properties of gases and liquids (4th ed.)". McGraw-Hill.

[3] Bader, A., Keller, P., Hasse, C., 2013, International Journal of Heat and Mass Transfer, 64, pp. 547-558.

[4] Keller, P., Knorsch, T., Wensing, M., Hasse, C., 2015, International Journal of Heat and Mass Transfer, 84, pp. 497-510.

[5] Camm, J., Stone, R., Davy, M., Richardson, D., 2015, SAE Paper 2015-01-0924.

[6] Law, C. K., 1976, Combustion and Flame, 26, pp. 219-233.

[7] Ra, Y., Reitz, R. D., 2009, International Journal of Multiphase Flow, 35, pp. 101-117.

[8] Ebrahimian, V., Habchi, C., 2011, International Journal of Heat and Mass Transfer, 54, pp. 3552-3565.

[9] Kitano, T., Nishino, J., Kurose, R., Komori, S., 2014, Fuel, 136, pp. 219-225.

[10] Sirignano, W. A., 2010, "Fluid dynamics and transport of droplets and sprays (2nd ed.)". Cambridge University Press.

[11] Wen, C. C., Tu, C. H., 2007, Fluid Phase Equilibria, 258, pp. 131-139.

[12] Xu, Z., Yi, J. W., Wooldridge, S., Reiche, D., Curtis, E. W., Papaioannou, G., 2009, SAE Paper 2009-01-1493.

[13] Yang, W., Xia, J., Wang, X. Y., Wan, K. D., Megaritis, A., Zhao, H., 2021, International Journal of Heat and Mass Transfer, 168, article no. 120876. 